\title{
Pediatric Brain tumors in Southeast Nigeria; An 8- year prospective longitudinal cohort study
}

Enoch Uche ( $\square$ kechyenny@yahoo.com )

University of Nigeria Faculty of Medical Sciences https://orcid.org/0000-0002-1562-6650

Nkechi Judith Uche

UNN Ituku Ozalla

\section{Obinna V Ajuzieogu}

University of Nigeria Faculty of Medical Sciences

\section{Dubem Amuta}

University of Nigeria Teaching Hospital

\section{ephraim Onyia}

University of Nigeria Teaching Hospital

\section{Chris Eke}

University of Nigeria Faculty of Medical Sciences

John Okafor

University of Nigeria

John-Kennedy Emejulu

Nnamdi Azikiwe University Teaching Hospital

\section{Wilfred Mezue}

University of Nigeria Faculty of Medical Sciences

\section{Matt Ryttlefors}

Akademiska sjukhuset

\section{Magnus Tisell}

Sahlgrenska universitetssjukhuset

\section{Research article}

Keywords: Pediatric brain tumors, microneurosurgery, radiotherapy, survival

Posted Date: September 4th, 2019

DOI: https://doi.org/10.21203/rs.2.13963/v1

License: (c) (i) This work is licensed under a Creative Commons Attribution 4.0 International License. Read Full License 


\section{Abstract}

Background: Pediatric brain tumors (PBT's) from previous studies are associated with poor outcomes in our subregion.

Methods. An 8 -year single center prospective study. All cases investigated with neuroimaging and treated were enrolled. Data was analyzed with SPSS (Inc) Chicago IL, USA version 23. Chi Square test, One-way Anova and confidence limits were used to evaluate associations using the $95 \%$ level of significance. Patients were followed up for a range of 1 to 7.5 years with a mean of $4.9 \pm 1$.3years. Ethical approval was obtained for our study.

Results: 95 patients were enrolled, 84 satisfied the study criteria. There were 45 males and 39 females, M: $\mathrm{F}=1.1$. The mean age was $9.9 \pm 2.7$ years $95 \% \mathrm{Cl}$ with a range of 9 months to 16 years. The most common symptom was headache for supratentorial lesions (73\%) and gait disturbance (80.2\%) for infratentorial lesions. More tumors were supratentorial in location (45(54.2\%), while 33(37.1\%) were infratentorial. Craniopharyngiomas $(n=19)$, medulloblastomas $(n=17)$ and astrocytomas $(n=11)$ were the most common tumors. Hemoglobin genotype(AA and AS) had some influence on tumor phenotype, Odds ratio 8.9 and 3.3 for medulloblastoma and craniopharyngioma. 69 cases were microsurgically resected while 14 patients were treated with radiotherapy alone. The 30 -day mortality for operated cases is $7.9 \pm 1.3 \%$. Overall 1-year and 5 -year survival was $67.9 \%$ and $53.6 \%$ respectively. Survival rates varied among treatment groups $(\mathrm{X} 2=8.9, \mathrm{P}=0.017)$.

Conclusion: Survival profile in this series suggests some improvement in comparison to previous studies from our region.

\section{Background}

Pediatric brain tumors (PBT's) in general currently constitute a great challenge confronting pediatric oncology units in many countries. [4] They contribute a significant proportion to the

overall morbidity and mortality profile of pediatric tumors worldwide. [4]The poor outcome

associated with PBT in the developing world is the result of a complex interplay of pathobiological

factors and environment-dependent care constraints presenting a relentless clinical challenge. Our

previous study presented a retrospective analysis of the outcomes prior to establishment of 
multidisciplinary oncology boards. ${ }^{2}$ In our index study we have observed and analyzed the presentation and outcome of a prospective cohort of pediatric patients with brain tumors who

received multidisciplinary care with microneurosurgery as the dominant treatment paradigm.

\section{Methods}

An 8-year single institution prospective observational study of pediatric patients with brain tumors

who received multidisciplinary care and were followed up between January 2010 and

December 2017. Our unit is the apex referral neurosurgical center in Southeast Nigeria serving a

large catchment population of up to 60 million people. Consent was obtained from caregivers

before enrollment of patients. Ethical approval for the study was obtained from the IRB of our

hospital. Our pediatric brain tumor patients receive multidisciplinary care with microneurosurgery

and radiotherapy as the main treatment modalities. A multidisciplinary oncology board review is

a component of our current patient care plan. Those with neuroimaging confirmed brain stem

gliomas or marker positive ICGCT are treated by radiotherapy without microsurgical resection.

We currently do not have a hospital chemotherapy protocol for pediatric brain tumors. Radiotherapy treatment plans are case based and discussed at oncology board reviews.

\section{Inclusion criteria:}

Patients aged 16 years or less with brain tumors evaluated clinically, verified by neuroimaging and treated either with microneurosurgery or radiotherapy alone or in 
combination. Microneurosurgical resection status was defined as total microneurosurgical resection (TMNR) for complete resection verified by post-operative MRI within 48 hours of surgery or subtotal microneurosurgical resection (SMNR) for incomplete resection which is $>$

90\% tumor volume resection.

All patients with histopathological confirmation

All neuroimaging verified brainstem tumors or intracranial germ cell tumors (ICGCT) with tumor

marker seropositivity with or without histopathological proof.

\section{Exclusion criteria.}

All cases without histopathological confirmation except brainstem or ICGCT's.

Pediatric brain tumors operated in other facilities but referred to our center for radiotherapy $(\mathrm{n}=3)$

All cases lost to follow up(n=8)

\section{Data Acquisition and analysis}

The age, sex and schooling profiles of the patients as well as the genotype, main presenting symptoms and signs were recorded as well as the neuroimaging (brain MRI diagnosis),

serum tumor marker profiles, treatment modality received such as surgery, surgery with radiotherapy or radiotherapy alone. All cases were followed up for a range of 1 to 7.5 years (mean

of $4.9 \pm 1.3$ years $(95 \% \mathrm{CI})$ with brain MRI scan 6 monthly for 2 years and yearly afterwards. 30-day mortality, 1 -Year and 5-Year survival rates were also evaluated. Analysis of general and

subgroup outcomes among histopathological variants and treatment modalities was performed as 
well. The influence of brain tumors and their treatment on schooling was evaluated and provided

an indirect assessment of the effect of PBT on cognitive function. All data were analyzed with

SPSS (Inc) Chicago IL, USA version 23. Confidence limits, Chi square test and One-way Anova

were used to evaluate associations between groups and subgroups at the $95 \%$ level of significance.

\section{Results}

95 patients were enrolled, while 84 satisfied the study criteria. Of 341 brain tumors treated in our center 
during this period, pediatric tumors account for 24.6\%. There were 45 males and 39 females, $\mathrm{M}: \mathrm{F}=1.1$.

Their ages ranged from 9 months to 16 years with a mean of $9.9 \pm 2.7$ years $(95 \% \mathrm{CI})$. Case frequency per

year ranged from 5 to 19 with a mean of $10.5 \pm 1.7$. Table 1 The most common symptom was headache for

supratentorial lesions (73\%) and unsteadiness of gait (80.2\%) for infratentorial lesions. Seizures was a

presenting symptom in 37(44\%) patients. In 45(53.6\%) patients, tumors were supratentorial in location,

while in 33(39.3\%) and 6(7.1\%) patients respectively, they were infratentorial and trans tentorial.

Craniopharyngiomas $(n=19)$, medulloblastomas $(n=17)$ and astrocytomas $(n=11)$ were the most common

tumors. However when brain stem gliomas, a clinical group that are usually not biopsied $(n=9)$ as well as

oligodendroglia's $(n=2)$ are added to the astrocytomas, gliomas $(n=22,(26.2 \%)$ are the most common tumor

subgroup in our series. The most common tumors encountered among the age subgroupings is shown in

TABLE 1 and TABLE 2. Dysembryoplastic Neuroectodermal Tumors (DNETS) and low grade

astrocytomas were the only tumors seen in children below 2 years of age, ependymomas and

medulloblastomas were the most common tumor subtypes among age subgroups 3-5, 6years respectively.

Craniopharyngiomas were the most common tumors among 9 to 11 and 12 to14- year age group and also

was the most common tumor among surgically treated patients in this series. Low grade gliomas were the 
dominant tumors among 15-16-year olds. 43 patients harbored WHO grade I tumor, while 3,2 and 21

patients respectively harbored WHO grades II, III and IV tumors. Analysis of the variability between age

groups and tumor type showed was significant using one -way Anova $(\mathrm{F}=6.4 \mathrm{DF} 1=2$, $\mathrm{DF} 2=12, \mathrm{P}=0.013)$.

70(83\%) patients were of Hb AA Genotype, while 7(8.3\%) had AS genotype, while 2(2.38\%) had SS

genotype. In 5 patients $\mathrm{Hb}$ genotype was not determined. Among $\mathrm{Hb} \mathrm{AA}$ patients, medulloblastoma was

the most common tumor $(n=15$, Odds ratio=8.9), Apart from medulloblastoma, the overall distribution of

other tumors among patients with $\mathrm{Hb}$ AA genotype was however did not vary significantly using one-way

Anova test. $(\mathrm{F}=2.33, \mathrm{P}=0.713)$.

There was a preponderance of craniophayngiomas among Hb AS subgroup $\left(n=5, X^{2}=9.7\right.$, $\mathrm{P}=0.002$, Odds ratio $=3.3)$ and low grade astrocytomas among sicklers $(\mathrm{n}=2, \mathrm{P}=0.000)$. TABLE 1.

69 cases were microsurgically resected. Of 39 patients treated with surgery alone, 25 of them had

TMNR, while 14 had SMNR. 30 patients had SMNR with radiotherapy. In all, 41 resected tumors had

adjuvant radiotherapy, while 14 patients were treated with radiotherapy as primary treatment for

brainstem gliomas (9 cases) or marker positive intracranial germ cell tumors (ICGCT $n=5$ ) TABLE 3. 37

cases of tumor associated hydrocephalus (TAH) received preoperative cerebrospinal fluid $(\mathrm{CSF})$

diversion with ventriculoperitoneal shunt (VPS), while 10 received endoscopic third ventriculostomy 
(ETV), 4 patients had a temporary External Ventricular Drainage (EVD). Three EVDs were discontinued

after tumor resection while one EVD was replaced with VPS. For craniopharyngiomas, 3 cases had

Ommaya reservoir inserted for cyst aspiration following recurrence. For intracranial germ cell tumors, the

presence a pineal region or suprasellar tumor with raised serum tumor markers (alpha fetoprotein and $\beta$ -

HCG) was diagnostic of malignant intracranial germ cell tumor (ICGCT) and this was seen in $5(6.0 \%)$

patients TABLE 2. We found a high CSF to blood gradient of $\alpha$-fetoprotein and $\beta$-HCG in three patients.

The CSF to blood gradient ranged from $2.7 \pm 0.9(95 \% \mathrm{CL})$ for alpha fetoprotein to $3.5 \pm 0.3(95 \% \mathrm{CL})$ for $\beta$ -

HCG among ICGCTs.

The treatment complication rates as well as 30-day post-operative mortality is shown (Table 1). The mean

complication rate is $13.9 \pm 3.7 \%$, while the mean 30 -day operative mortality rate is $7.9 \pm 1.3 \%$, The long-

term survival profiles are shown in TABLE 3. Survival rates varied both among treatment subgroups.

$\mathrm{X}^{2}=8.9, \mathrm{P}=0.017$. Overall 1-year and 5-year survival generally was $67.9 \%$ and $53.6 \%$ respectively. The

influence of paediatric brain tumors and their treatment on school attendance and performance is shown

in table 4 . Only $15(18.5 \%)$ patients completed primary or secondary education and those who received

surgical treatment only were more likely to complete their schooling $\mathrm{X}^{2}=4.94, \mathrm{P}=0.015$. 


\section{Discussion}

From our study, the mean annual incidence of pediatric brain tumors receiving specialized care

in our center is 10.5 cases. Children accounted for $24.6 \%$ of all brain tumors treated in our center

during this period. Our hospital is a 1000- bed tertiary health facility; the largest multispecialty

hospital in the southeast region of our country endowed with a robust workforce in paediatric

neurosurgery. The annual incidence appears low; however, it may reflect constraints in accessing

care by patients generally and pediatric brain tumor patients specifically. Some of the constraints

have been studied extensively [23, 30]. At $9.9 \pm 2.7$ years, the mean age of occurrence of pediatric

brain tumors is similar to that from a previous study performed by the lead author of this paper

and other researchers from another region of our country [30]. as well as a recent retrospective 
study from a center in our subregion with a smaller sample size [22]. In Morocco, a North African

country, a large epidemiological study found a mean age of 9.3years [11]. A slight male preponderance found in our study is similar to some previous studies both from our country and

elsewhere $[4,22,23,30]$. Headache was the most common presenting features among supratentorial tumors while unsteadiness of gait occurred more frequently among infratentorial

tumors. 53.6\% of tumors in our index series occurred in the supratentorial compartment.

Although the finding above is supported by another report from our sub region [4], it differed from

reports from other regions in our country [1, 23,30] as well as previous studies in other parts of

the world $[4,11,33]$. This may suggest the existence of geographical variations in the topographical

distribution of brain tumors which in the view of some authors may also be influenced by age $[3$,

15]. Craniopharyngioma $(\mathrm{n}=19)$ is the most common tumor among histopathologically confirmed

cases in our series followed by medulloblastoma TABLE 2. However, when brain stem gliomas $(n=9)$ and oligodendrogliomas $(n=2)$ are included, gliomas $(n=22)$ will represent the most

common subgroup. Our previous study showed that medulloblastoma and astrocytomas were the

most common tumors, while craniopharyngiomas were the third most common tumor subtype

[30]. An epidemiological study from Morocco, a country in the North of Africa, reported medulloblastoma as the most common tumor followed by low grade astrocytoma, while 
craniopharyngioma was the fifth most common tumor subtype [11]. We found a significant age

group bias in the occurrence of brain tumors among our cohorts $(F=16.4, \mathrm{df} 1=2$, df $2=12$,

$\mathrm{P}=0.013)$ TABLE 1. Low grade Astrocytoma and DNETS were seen in patients below 2 years of

age. Some previous studies have also found a preponderance of astrocytomas in under -2 children

$[6,8]$. Ependymomas were predominant among 3 to 5-year age group, while medulloblastomas

were most common among age group 6 to 8 years respectively. Previous studies have also reported

age group variability in the occurrence of ependymomas and medulloblastomas $[5,17,20]$, as well

as craniopharyngiomas which from our series is the most prevalent tumor between ages 9 and 14

years. A previous study found a higher incidence of craniopharyngiomas among black children in

the United States of America [29]. The findings of our study seem to highlight as well this racial

and geographical predisposition to pediatric brain tumors or may reflect a changing trend more so

with the predominance of craniopharyngiomas in our series $(22.6 \%)$ when compared to previous

studies from other centers in our country [22, 23, 30], and elsewhere, some of which reported a

higher frequency of occurrence of astrocytomas and medulloblastomas $[3,9,10]$.

Low grade gliomas were predominant among adolescents between 15 and 16 years. We found an

association between hemoglobin genotype and brain tumors. For instance, medulloblastomas 
occurred more commonly among patients with $\operatorname{HbAA}(n=15$, Odds ratio=8.9), while

craniopharyngiomas occurred mostly in $\mathrm{HbAS}$ patients (Odds ratio $=3.3, \square^{2}=9.7, \mathrm{P}=0.002$ ). Moreover, both patients with $\mathrm{Hb}$ SS in our series had low grade astrocytomas, $(\mathrm{n}=2$, $\mathrm{P}=0.000)$.

Our findings are supported by a previous study which reported 3 cases of astrocytoma among 47 sicklers with cancer [27]. To our knowledge, our study remains to date the largest

prospective study reporting an association between hemoglobin genotype and the predisposition

to specific brain tumor phenotypes. We do not know the extent to which our observations represent the salient relationship between hemoglobin genotype and brain tumor profiles in children. A multicenter study is necessary to further verify this association especially within sub-

Saharan countries which alongside the Indian subcontinent currently harbor over $90 \%$ of the

global burden of sickle cell disease [24]. Such a study may generate scientific evidence to suggest

a review of the current risk factor associations of PBT's.

We applied microneurosurgical resection to most cases $(n=69)$. Total microneurosurgical resection (TMNR) alone was the treatment modality in $52.5 \%$ of surgically treated cases. Of all

microsurgically resected tumors, 43 cases were WHO Grade I, 3 were Grade II, 2 were Grade III

and 21 were Grade IV tumors. Surgery provides a direct and effective treatment path for brain

tumors and the extent of resection correlates with overall outcome [12, 19, 33]. From our series,

those who received TMNR had the best survival profile Table 3 and also the best school 
performance Table 4. However, majority of brain tumors in paediatric patients grow near functionally eloquent sites presenting a significant challenge to preserving them during surgery

[31]. Intraoperative neuronavigation and brain function mapping facilitate tumor resection and

help in preserving eloquent brain loci during surgery. ${ }^{24}$ We currently do not have these parameters

in our unit and hence rely on microneurosurgical techniques. We believe the absence of neuronavigation and intraoperative brain mapping may be a significant limiting factor to performance of TMNR and may invariably play some role in lowering the survival profile of our

patients.

The role of radiotherapy in the treatment of pediatric brain tumors is well recognized [16, 25].

However, from our series, patients treated with radiotherapy had poorer overall survival (Table 3)

as well as school performance. Poor school outcomes with radiotherapy may partly reflect the

reality that more high-grade tumors with intrinsic propensity to poorer outcomes are treated with

this modality including brainstem gliomas. However, the long- term sequelae of radiation therapy

among paediatric brain tumor survivors especially its harmful effect on brain vasculature and

neurocognitive functions have been previously reported $[2,21]$. Despite these sequelae, radiotherapy together with cerebrospinal fluid diversion remain the mainstay for treatment of brain

stem gliomas which constitute $10.6 \%$ of pediatric brain tumors in our series[32]. Radiation 
treatment for brainstem gliomas in our series was associated with 40\% and 0\%, 1 -Year and 5 -

Year survival rates respectively which compares favorably with a report from a recent Asian

Study[32]. We also treated serum marker positive ICGCT's with radiotherapy and the 1 year and

5-year survival rates for ICGCT from our series is $60 \%$ and $40 \%$ respectively which compares

with some previously reported outcomes for non germinomatous germ cell tumors [13, 32].

From our index study, marker positive ICGCT constitutes $6 \%$ of all paediatric brain

tumors. The use of generous radiation dose for tumor bed and ventricular space with or without

craniospinal irradiation and chemotherapy is considered a vital component of the standard treatment protocol for germ cell tumors [18, 32]. Treatment complications and 30-day mortality

rates varied with tumor phenotype. Medulloblastoma was associated with the highest rate of post-

op complications and 30-day mortality TABLE 1, while Ependymomas, craniopharyngiomas and

low-grade gliomas were associated with lower rates.

Patients overall survival (OS) also varied with tumor phenotype as well as treatment modality

TABLE 1. Craniopharyngiomas, meningiomas, low grade gliomas, and ependymomas

were associated with the best survival, while brainstem gliomas and medulloblastomas had the worst survival. Patients who received TMNR had the best survival of any treatment subgroup,

while surgically treated patients survived better than those treated with radiation $\left(\square^{2}=8.9\right.$, $\mathrm{P}=0.017$ ) TABLE 3. Although the overall 1-year and 5-year survival rates of $67 \%$ and $52 \%$ 
respectively from our current study compare poorly to those from centers in the developed world $[14,26]$, they however represent an improvement when compared to our previous work as well as a recent study from our sub region [22, 30]. In our previous study, the 1year and

5-Year survival rates were $53 \%$ and $47 \%$ respectively [30]. We believe this improvement is the

result of multiple factors, including the establishment of a multidisciplinary oncology center which

has an active radiotherapy unit equipped with a linear accelerator as well as increase in the number

of neurosurgeons with experience in paediatric neurosurgery. Furthermore, an increase in awareness and improved referral practices may partly account for the overall positive survival shift

in this study, which more so is significantly more experienced among those treated with TMNR.

From our study, PBT's negatively affect school attendance and performance, table 4 and the treatment modalities are also associated with variable influence on school performance.

Our findings are quite significant because PBT survivors are often excluded from studies evaluating childhood cancer survivors, due in part to concerns on the impact of cognitive limitations on the validity of assessment [26. 34].

To our knowledge, this is the first study evaluating school performance among survivors of PBT in our region and therefore provides the reference for further studies on this subject. More

clinical studies and collaborations are needed between centers treating PBT's within the sub-

Saharan region so as to further elucidate the variations in presentation, treatment practices, as well 
as evaluate relevant potentially significant associations including hemoglobin genotype. This will

help us to summate the outcome of pediatric brain tumors within the region.

\section{Limitation Of The Study}

Our study reports a one- center experience. As a result, it is invariably exposed to the 'tip of the

iceberg phenomenon' that negatively profiles single center studies from tertiary referral centers.

We did not report an experience with chemotherapy for treatment of PBT's due to a lack of protocol for PBT chemotherapy in our center

\section{Conclusions}

Craniopharyngiomas are among the most common tumors in our series of PBT's.

Hemoglobin genotype may have an influence on PBT phenotype in our environment. TMNR is

associated with better overall survival and school performance profiles. Overall survival is improved in our study when compared to previous studies from our subregion. Multicenter collaborations are however required to better define the characteristics of PBT's.

\section{Declarations}

Ethics approval: This Study was approved by the IRB of University of Nigeria Teaching Hospital

Enugu Nigeria with ethical reference number: NHREC/05/01/2008B-FWA00002458-

1RB00002323. Both verbal and written consent for participation was obtained from all caregivers.

Consent to Publish: Consent was also obtained from all the care givers for publication of the

study results. 
Availability of data and materials: The data from this study are not sensitive and we do not require help with data management. The study materials are available for review if required.

Competing Interest Statement: We have no competing interest to declare

Funding: We did not receive any external funding for our work.

Authors' Contributions: All the authors contributed significantly to the study and all have given

final approval for submission and publication of the manuscript.

Acknowledgement: We wish to appreciate the invaluable contributions of Dr Okpara and Dr Guga, both senior registrars in our unit who facilitated this work in many salient ways. We also

wish to acknowledge our hospital's neurosurgical ward nursing staff for their invaluable contribution to the care of our pediatric patients with brain tumor.

\section{Abbreviations}

\begin{tabular}{|l|l|}
\hline \multicolumn{2}{|l|}{ Abbreviations } \\
\hline PBT's & Pediatric Brain Tumors \\
\hline SPSS & Statistical Package for Social Sciences \\
\hline ICGCT & Intracranial Germ Cell Tumors \\
\hline IRB & Institutional Review Board \\
\hline TNMR & Total Microneurosurgical Resection \\
\hline SMNR & Subtotal Microneurosurgical Resection \\
\hline MRI & Magnetic Resonance Imaging \\
\hline DNETS & Dysembryoplastic Neuroectodermal Tumors \\
\hline WHO & World Health Organization \\
\hline Hb & Hemoglobin \\
\hline TAH & Tumor Associated Hydrocephalus \\
\hline CSF & Cerebrospinal Fluid \\
\hline ETV & Endoscopic Third Ventriculostomy \\
\hline EVD & External Ventricular Drainage \\
\hline VPS & Ventriculoperitoneal Shunt \\
\hline$\beta-H C G ~$ & Beta-Human Chorionic Gonadotropin \\
\hline
\end{tabular}




\section{References}

1. Adeloye A, Nottidge VA, Udi J. Craniopharyngiomas in Nigerian Children. Childs Nerv Syst. 1988;4:128134. 2. Avadiappan S, Morrison M, Jakary A, Felton E, Mueller S, Lupo J. Characterization of radiation Therapy Effects on Cerebral Vasculature in Pediatric Brain Tumors. Neuro- Oncol 2018;20(Suppl 6): vi229. 3. Bauchet. L, Rigau V, Mathieu-Daude H, Fabbro-Peray P, Palenzuela G, Figarella - $\backslash$ Branger D, et al. Clinical epidemiology of childhood primary central nervous system tumors. J. Neurooncol 2009;92(1):8798. 4. Bleyer WA. Epidemiologic impact of children with brain tumors. Childs Nerv Syst 1999;15(1112):758-763. 5. Crawford JR, Macdonald TJ, Packer JR. Medulloblastoma in Childhood: new biological advances. Lancet Neurol 2007;6:1073-1085. 6. Furuta T, Tabuchi A, Adachi Y, Mizumatsu S, Tamesa N, Ichikawa T, Tamiya T, Matsumoto K, Ohmoto T. Primary Brain Tumors under 3 years. Brain Tumor Pathol. 1998;1:7-12. 7. Gregory K, Parker L, Craft AW. Returning to primary school after treatment for cancer. PediatrHematol Oncol 1994;11:105-109. 8. Ibrahim K, Appleton R. Seizures as the presenting symptom of Brain Tumors in Children. Seizure 2004;13108-112. 9. Kaatsch P, Rickert CH, Kuhl J, Schuz J, Michaelis J. Population-based epidemiologic data on brain tumors in German children. Cancer 2001;92(12):31-3164. 10. Kaderali Z, Lamberti-Pasculli M, Rutka JT. The Changing epidemiology of paediatric brain tumors: a review from the Hospital for Sick Children. Childs Nerv Syst. 2009 Jul;2(7):787-93. 11. Karkouri M, Zafad S, Khattab M, Benjaffar N, Elkacemi H, Sefiani S, Kettani F, Dey S Soliman AS. Epidemiologic Profile of Pediatric brain tumors in Morocco Childs Nerv Syst. 2010;26(8):1021-1027. 12. Khalil EM. Treatment of adults and children with medulloblastoma NCl, Cairo University experience. J. Egypt Natl Canc Inst 2008;20:175-186. 13. Kim JW, Kim WC, Cho JH, Kim DS, Shim KW, Lyu CJ, et al. A multimodal approach including craniospinal irradiation improves the treatment outcome of high risk intracranial nongerminomatous germ cell tumors. Intl J Radiat Oncol Biol Phys. 2012;84:625-631. 14. Kim JY, Park J. Understanding the Treatment Strategies of Intracranial Germ Cell Tumors: Focusing on Radiotherapy. J Korean Neurosurg Soc. 2015 May;57(5):315-322. 15. Koob M, Girard N. Cerebral Tumors: Specific features in Children. Diagnostic and Interventional Imaging 2014;95(10):965-983. 16. Knab B, Connell PP. Radiotherapy for pediatric brain tumors: when and how. Expert Rev Anticancer Ther. 2007:7(12 Suppl):S69-77. 17. MaksoudYA, Hahn YS, Engelhard HH. Intracranial Ependymoma. Neurosurgical focus 2002;13:e4. 18. Matsusani M. Japanese Pediatric Brain Tumor Study Group. Combined chemotherapy and radiation therapy for CNS germ cell tumors-The Japanese experience. J Neuroncol.2001:54:311316. 19. McGirth MJ, Chaichana KL, Attenello FJ, Weingart JD, Than K, Burger PC, Olivi A, Bram H, Quinones-Hinojosa A. Extent of Surgical Resection is Independently Associated with Survival in Patients with Hemispheric Infiltrating Low Grade Gliomas. Neurosurg 2008;63(4):700-708. 20. Modha A, Vassilyadi M, George A, Kuehn S, Hsu E, Ventureyra EC. Medulloblastoma in children-the Ottawa experience. Childs Nerv Syst. 2000 Jun;16(6):341-350. 21. Mulhern RK, Hancock J, Fairclough D, Kun L. Neuropsychological status of children treated for brain tumors: A critical review and integrative analysis. Pediatric Blood and Cancer 1992.20(3):181-192. 22. Ndubisi CA, Ohaegbulam SC, Ejembi GO. Paediatric Brain tumors managed in Enugu, Southeast Nigeria: Review of one center experience. Niger Postgrad Med J. 2018;25(3):186-190. 23. Ogun GO, Adeleye AO, Babatunde TO, Ogun OA, Salami A, Brown BJ, Akang E. Central Nervous System Tumors in Ibadan, Nigeria: a histopathologic study. Pan African Medical Journal 
2016; 24:34. 24. Piel FB, Hay SI, Gupta S, Weatherall DJ, Williams TN. Global burden of sickle cell anaemia in children under five,2010-2050: modelling based on demographics, excess mortality, and interventions. PloS Med.2013 Jul;10(7).e1001484. 25. Pollack IA. Brain tumors in Children. NEJM. 1994;331:100-107. 26. Ries LAG, Eisner MP, Kosary CL et al. SEER Cancer Statistics Review, 19731999.Bethesda MD, National Cancer Institute 2002.http://seer.cancer.gov/csr/1973-1999/ 27. Schultz WH, Ware RE. Malignancy in Patients with Sickle Cell Disease. American Journal of Hematology 2003;74:249-253. 28. Steinbok P. Mangat JS, Kerr JM, Sargent M, Suryaningtyas W, Singhal A, Cochrane D. Neurological morbidity of surgical resection of pediatric cerebellar astrocytomas. Childs Nerv Syst 2013;29:1269-1275. 29. Stiller CA, Nectoux J. International Incidence of childhood brain and spinal tumors. Int J Epidemiol. 1994;23(3):458. 30. Uche EO, Shokunbi MT, MalomoAO, Akang EE, Lagunju I, Amanor-Boadu SD. Pediatric Brain Tumors in Nigeria: clinical profile, management strategies, and outcome. Childs Nerv Syst 2013 July; 29(7):1131-5. 31. Vabulas M, Kumar VA, Hamilton JD, Martinez JJ, Rao G, Sawaya R, Prabhu SS. Real- time-atlas-based stereotactic neuronavigation. Neurosurgery 2014;74:128-134. 32. Yoshida K, Sulaiman NS, Miyawaki D, Ejima Y, Nishimura H, Ishihara T, Matsuo Y, Nishikawa R, Sasayama T et al. Radiotherapy for brainstem gliomas in children and Adults: A single institution experience and literature review. Asia Pac J Clin Oncol. 2017 Apr;13(2):e153-e160. 33. Yu J, Shi W, Zhao R, Gao X, Li H. Epidemiology of brain tumors in children aged two and under:A 10-year single center study. Oncol Lett.2016 Apr;9(4):1651-1656. 34. Zebrack BJ, Gurney JG, Oeffinger K, Whitton J, Packer RG, Mertens A, Turk N, Castleberry R, Dreyer Z, Leslie L, Zelter LK. Psychological outcomes in Long-Term Survivors of Childhood Brain Cancer: A Report From the Childhood Cancer Survivor Study. J Clin Oncol 2004; 22(6):999-1006.

\section{Tables}

Due to technical limitations, tables are only available as a download in the supplemental files section

\section{Supplementary Files}

This is a list of supplementary files associated with this preprint. Click to download.

- supplement1.docx

- supplement2.doc

- supplement2.doc

- supplement4.doc 